Check for updates

Cite this: Chem. Commun., 2020, 56,8822

Received 15th May 2020,

Accepted 17th June 2020

DOI: $10.1039 / \mathrm{dOcc03490h}$

rsc.li/chemcomm

\section{A rapid synthesis of low-nanomolar divalent LecA inhibitors in four linear steps from D-galactose pentaacetate $\uparrow$}

\author{
Eva Zahorska, (DD abc Sakonwan Kuhaudomlarp, (D) ${ }^{d}$ Saverio Minervini, ${ }^{a}$ \\ Sultaan Yousaf, ${ }^{a}$ Martin Lepsik, (iD d Thorsten Kinsinger, ${ }^{a}$ Anna K. H. Hirsch, ID bce \\ Anne Imberty ${ }^{d}{ }^{d}$ and Alexander Titz (D) *abc
}

Chronic infections with Pseudomonas aeruginosa are associated with the formation of bacterial biofilms. The tetrameric $P$. aeruginosa lectin LecA is a virulence factor and an anti-biofilm drug target. Increasing the overall binding affinity by multivalent presentation of binding epitopes can enhance the weak carbohydrate-ligand interactions. Lownanomolar divalent LecA ligands/inhibitors with up to 260-fold valency-normalized potency boost and excellent selectivity over human galectin-1 were synthesized from D-galactose pentaacetate and benzaldehyde-based linkers in four linear steps.

Pseudomonas aeruginosa has been classified as a priority-1 pathogen by the World Health Organization due to its high antimicrobial resistance and the lack of new drugs to treat multidrug-resistant strains. ${ }^{1}$ New strategies against these bacterial infections are being explored to overcome the current antimicrobial-resistance crisis. ${ }^{2}$ The so-called anti-virulence therapy aims to neutralize bacterial virulence factors instead of increasing the selection pressure imposed by targeting essential cellular functions with antibiotics and thereby circumvents the advent of new resistances whilst preserving commensal bacteria., ${ }^{3,4}$ This strategy is investigated for $P$. aeruginosa infections by targeting its tetravalent lectins LecA and LecB. ${ }^{2,5,6}$ Both proteins are virulence factors regulated by quorum sensing, mediate bacterial host-cell adhesion and are essential structural components of $P$. aeruginosa biofilms. ${ }^{7-9}$ Whereas the best L-fucose/D-mannose-based LecB antagonists bind in the nanomolar range, monovalent $\mathrm{D}$-galactose-based LecA inhibitors only

\footnotetext{
${ }^{a}$ Chemical Biology of Carbohydrates, Helmholtz Institute for Pharmaceutical Research Saarland, Helmholtz Centre for Infection Research, 66123 Saarbrücken, Germany.E-mail: alexander.titz@helmholtz-hzi.de

${ }^{b}$ Deutsches Zentrum für Infektionsforschung (DZIF), Standort HannoverBraunschweig, 38124 Braunschweig, Germany

${ }^{c}$ Department of Pharmacy, Saarland University, 66123 Saarbrücken, Germany

${ }^{d}$ Université Grenoble Alpes, CNRS, CERMAV, 38000 Grenoble, France

${ }^{e}$ Drug Design and Optimization, Helmholtz Institute for Pharmaceutical Research Saarland, Helmholtz Centre for Infection Research, 66123 Saarbrücken, Germany $\dagger$ Electronic supplementary information (ESI) available. See DOI: 10.1039/ docc03490h
}

reach binding affinities in the mid to low micromolar range. ${ }^{10-15}$ In Nature, the rather weak lectin-carbohydrate binding is often overcome by increasing valency, and thus enhancing apparent affinity. ${ }^{16,17}$ Likewise, a boost in target-binding affinity was achieved with multivalent inhibitors of LecA and LecB. ${ }^{6,18}$ Since LecA is a tetramer and pairs of binding sites are geometrically favorably oriented, simultaneously binding divalent inhibitors can boost binding affinity through favorable binding entropy. ${ }^{19,20}$ Notably, Pieters and co-workers have developed divalent LecA inhibitors based on complex and rigid repeating units of carbohydratetriazole spacers with potent binding affinities ranging from 12 to $220 \mathrm{nM},{ }^{21-23}$ while a divalent inhibitor with a more flexible linker reaches an affinity of $80 \mathrm{nM}^{24}$ In another report, an oligoprolinespaced digalactoside bound to LecA with $K_{\mathrm{d}}$ of $71 \mathrm{nM}^{25}$

In this work, we aimed to develop divalent LecA ligands with a focus on drug-like properties, synthetic accessibility and linker simplicity enabling future lead optimization. Spacer length and flexibility are important factors contributing to the overall potency of multivalent inhibitors. ${ }^{6}$ An optimized linker connecting two neighboring binding sites within one LecA tetramer and avoiding unwanted cross-linking between different LecA tetramers is desired (Fig. 1a). $\beta$-Linked aryl aglycons increase the binding strength of galactosides to LecA by establishing $\mathrm{CH}-\pi$ interactions with His50. ${ }^{15}$ The co-crystal structure of LecA with phenyl $\beta$-D-galactoside (PDB code: 5d21) showed possible growth vectors in meta- and para-position at the phenyl aglycon (Fig. 1b). ${ }^{26}$

Protein-templated dynamic combinatorial chemistry (DCC) is an elegant method for the identification of potent ligands from a combinatorial library of building blocks with suitable linking chemistry in presence of a given protein. ${ }^{27,28}$ To apply this method to LecA, we introduced hydrazides at the para- or meta-position of phenyl $\beta$-D-galactoside in order to allow for acylhydrazone formation in DCC. For this purpose, we chose two galactoside building blocks with meta- or para-attached hydrazides, $1 m$ and $1 p$, and varied linker length, rigidity and number of rotatable bonds by systematically increasing the number of methylene units in the corresponding benzaldehyde 


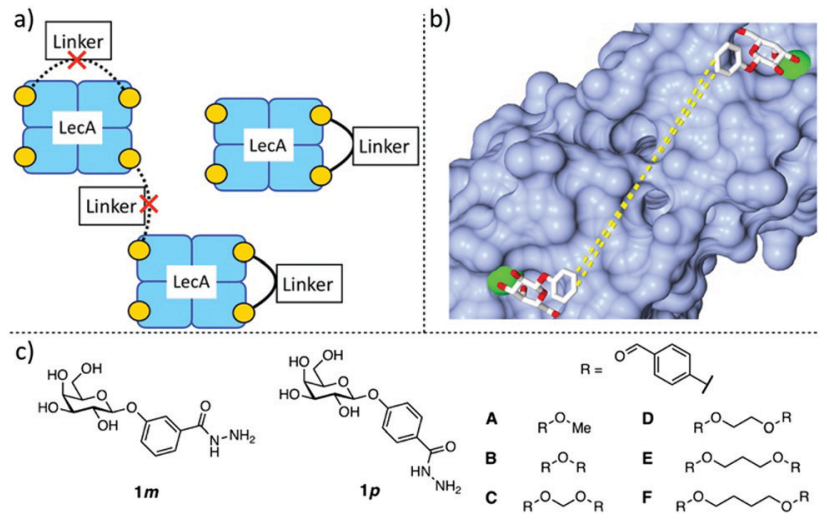

Fig. 1 Design of divalent LecA inhibitors accessible through a short synthetic route with acylhydrazone coupling chemistry. (a) Possible binding modes of divalent LecA inhibitors with desired linkage for two adjacent binding sites. (b) The crystal structure of phenyl $\beta$-D-galactoside in complex with LecA (pdb code: $5 \mathrm{~d} 21)$ and distances between two ligands within one pair of binding sites in LecA (from meta to meta: $23 \AA$, from para to para: $25 \AA$ ). (c) Building blocks of LecA inhibitors: $m / p$-hydrazinecarbonylphenyl $\beta$-D-galactopyranoside $(\mathbf{1} \mathbf{m}, \mathbf{1 p})$ and benzaldehydes $\mathbf{A}-\mathbf{F}$. Bis-benzaldehyde linkers $\mathbf{B}-\mathbf{F}$ with systematic variation of length and number of rotatable bonds to optimize distance and flexibility.

spacers B-F. The corresponding monovalent control A was included (Fig. 1c).

Benzaldehydes $\mathbf{A}$ and $\mathbf{B}$ were commercially available and bis-benzaldehydes $\mathbf{C - F}$ were obtained in one step using 4hydroxybenzaldehyde in a double nucleophilic substitution reaction on aliphatic $\alpha, \omega$-di-halogenated $\mathrm{C} 1-\mathrm{C} 4$ hydrocarbons under microwave irradiation (Scheme 1). Lewis acid-promoted glycosylation of methyl meta- or para-hydroxybenzoate with $\beta$-D-galactose pentaacetate (2) yielded glycosides $3 m$ and $3 p$ in $69 \%$ and $47 \%$ yield, respectively. Removal of the acetates under Zemplén conditions gave galactosides $\mathbf{4 m}$ and $\mathbf{4 p}$ quantitatively. Subsequent ester hydrazinolysis resulted in hydrazides $1 m$ and $1 p$ in very good yields. These building blocks were then used in DCC reactions in presence

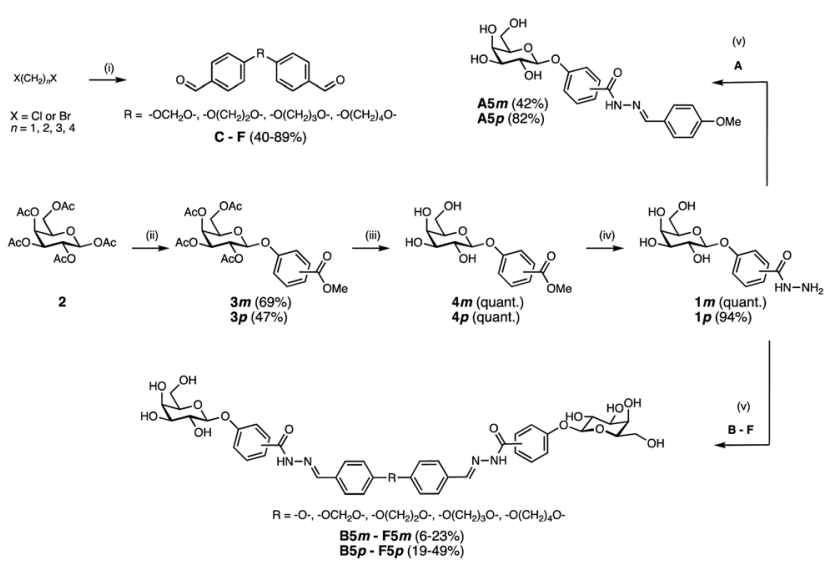

Scheme 1 Synthesis of divalent LecA ligands and their monovalent analogs. Reagents and conditions: (i) 4-hydroxy benzaldehyde, $\mathrm{K}_{2} \mathrm{CO}_{3}, \mathrm{DMF}$, $70{ }^{\circ} \mathrm{C}$, microwave, 3-10 h; (ii) methyl $\mathrm{m} / \mathrm{p}$-hydroxybenzoate, $\mathrm{BF}_{3} \cdot \mathrm{Et}_{2} \mathrm{O}$, $\mathrm{CH}_{2} \mathrm{Cl}_{2}, \mathrm{O}{ }^{\circ} \mathrm{C}$ - r.t., o.n.; (iii) $\mathrm{NaOMe}, \mathrm{MeOH}$, r.t., o.n.; (iv) $\mathrm{NH}_{2} \mathrm{NH}_{2} \cdot \mathrm{H}_{2} \mathrm{O}$, $\mathrm{MeOH}, 70{ }^{\circ} \mathrm{C}$, o.n. (v) formic acid, DMSO, r.t., 4 h, for D5m: DMSO/MeCN, for F5m: $\mathrm{H}_{2} \mathrm{O} / \mathrm{MeCN}$. of LecA: since the addition of LecA to the library caused precipitation, all divalent molecules were individually synthesized in absence of protein. Acylhydrazone formation of aldehydes A-F with excess hydrazide $1 m$ or $1 p$ under acidic conditions yielded the mono- and divalent LecA ligands A5m-F5m and A5p-F5 $p$. The reduced solubility of the meta-series compared to the para-series and more difficult purifications could explain the lower yields despite nearly quantitative turnover during the individual reactions.

All synthesized galactosides, A5m-F5m and A5p-F5p, were then analyzed in the previously established competitive LecA binding assay based on fluorescence polarization (Fig. 2). ${ }^{11}$ Monovalent meta-ligand A5m $\left(\mathrm{IC}_{50}=21.6 \pm 4.5 \mu \mathrm{M}\right)$ was twice as potent as its para-isomer A5p $\left(\mathrm{IC}_{50}=55.5 \pm 4.4 \mu \mathrm{M}\right)$. The divalent ligands B5m-F5m and B5p-F5p showed a very similar profile in the competitive binding assay with very similar $\mathrm{IC}_{50}$ values in the single-digit micromolar range and a very steep Hill slope of the fit. These observations are likely a result of reaching the lower assay limit since the low affinity of the fluorescent primary ligand $\left(K_{\mathrm{d}}=7.4 \mu \mathrm{M}\right)$ required a relatively high LecA concentration of $20 \mu \mathrm{M}$. Therefore, ligand affinities with orders-of-magnitude higher potencies than the primary competitively displaced ligand cannot be reliably determined.

To overcome the competitive binding assay's limitation, we analyzed all inhibitors in a direct LecA binding experiment using surface plasmon resonance (SPR). In case of the monovalent inhibitors $\mathbf{A 5 m}$ and $\mathbf{A 5} \boldsymbol{p}$, rapid changes in the binding response during the association and dissociation phases were observed, indicating fast association/dissociation kinetics of the monovalent inhibitors to immobilized LecA (Fig. 3a and b). Due to this fast association/dissociation behavior, $k_{\text {on }}$ and $k_{\text {off }}$ for their interaction with LecA could not be accurately determined and affinity analysis was performed instead of determining $K_{\mathrm{d}}$ at steady-state binding. In SPR, the monovalent ligands A5m and A5p have $K_{\mathrm{d}}$ values of $4.9 \pm$ 0.1 and $5.6 \pm 0.3 \mu \mathrm{M}$, respectively (Table 1 ). The binding affinities for those monovalent compounds were validated using isothermal titration microcalorimetry (ITC) as an orthogonal method and similar values to SPR data were obtained (ITC: $\mathbf{A 5 m} K_{\mathrm{d}}=2.7 \pm$ $1.3 \mu \mathrm{M}, \mathbf{A 5 p} K_{\mathrm{d}}=6.1 \pm 0.5 \mu \mathrm{M}$, Table 1 and Fig. S1, ESI $\dagger$ ). In both analyses, a meta-substitution of the phenyl aglycon in $\mathbf{A 5 m}$ resulted in higher affinities to LecA compared to the para-isomer A5p.

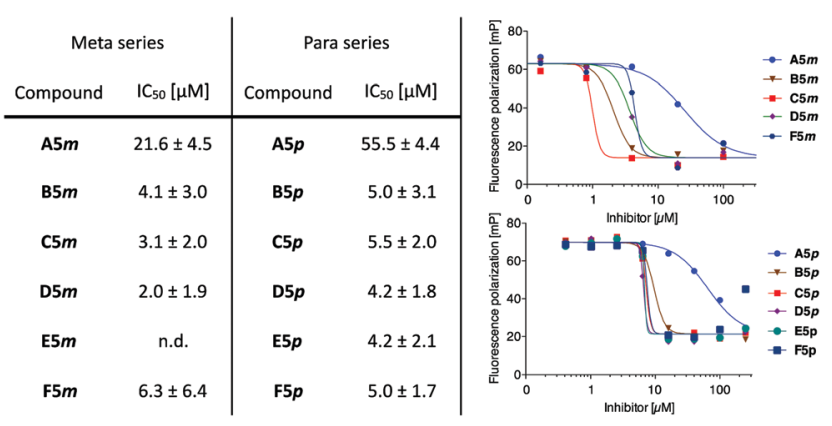

Fig. 2 Evaluation in a competitive binding assay. One representative titration is shown for each series (right) - steep titration slopes for divalent inhibitors indicate the lower assay limit was reached. Aver. and std. dev. from at least 3 independent titrations of triplicates each. n.d. = not determined. 

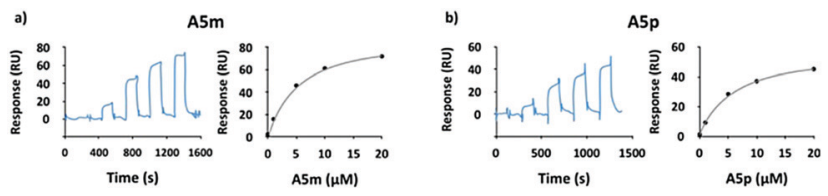

c)

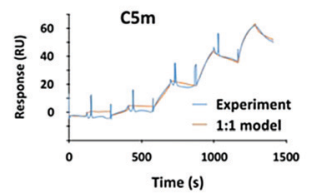

d)

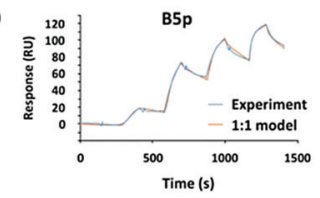

Fig. 3 SPR analyses of the interaction between the monovalent inhibitors (a) A5m and (b) A5p. The sensorgrams are shown on the left panel and the affinity analyses on the right. (c) Sensorgrams of the most potent divalent inhibitors from meta-series $(\mathbf{C 5 m})$ and (d) from para-series (B5p) at five different concentrations $(0,10,50,100,200 \mathrm{nM})$.

In contrast to the monovalent hydrazides, the SPR sensorgrams of the divalent inhibitors clearly indicated much slower association/dissociation of the compounds from LecA, demonstrating the benefit of divalent binding and enabling determination of kinetic parameters $\left(k_{\text {on }}\right.$ and $\left.k_{\text {off }}\right)$ as well as $K_{\mathrm{d}}$ (calculated from $k_{\text {off }} / k_{\text {on }}$, Fig. 3c, d and Fig. S2, ESI $\dagger$ ). The divalent inhibitors $\mathbf{B} 5 \boldsymbol{m}$-F5 $\boldsymbol{m}$ and B5p-F5p showed a strong increase in potency into the low nanomolar range (Table 1). In the para-series, compound B5p was the most potent ligand $\left(K_{\mathrm{d}}=10.8 \pm 1.0 \mathrm{nM}\right)$, with a 520fold increase (260-fold valency-corrected) compared to its monovalent congener A5p $\left(K_{\mathrm{d}}=5600 \pm 300 \mathrm{nM}\right)$. The divalent paraligands showed a slight decrease in potency with increasing spacer length, resulting from gradually decreasing $k_{\text {on }}$ and increasing $k_{\text {off }}$ (Table 1). In the meta-series, similar trends were absent. C5m with one central methylene unit had the optimal length and showed a $K_{\mathrm{d}}$ of $18.9 \pm 1.6 \mathrm{nM}$. Shortening or increasing spacer length resulted in reduced affinities and surprisingly the second longest compound E5m was the least efficient inhibitor $\left(K_{\mathrm{d}}\right.$ of $80.7 \pm$ $11.4 \mathrm{nM}$ ). Comparing across the meta-series, the $k_{\text {on }}$ values were surprisingly 2-4 times smaller for compounds C5m and E5m. The $k_{\text {off }}$ values were gradually increasing going from $\mathbf{C 5 m}-\mathbf{F} 5 \mathbf{m}$. The $k_{\text {off }}$ for B5m was 5-times higher than that of $\mathbf{C 5 m}$.

We then studied compound selectivity in binding experiments towards human galectin-1, a homodimeric lectin that specifically recognizes $\beta$-galactoside containing glycans such as Me- $\beta$-lactoside $\left(K_{\mathrm{d}}=187 \mu \mathrm{M}\right){ }^{29}$ The most potent inhibitors from the para- and meta-series, B5p and C5m, together with their respective monovalent counterparts (A5p and $\mathbf{A 5 m}$ ), were analyzed by SPR for their interaction with human galectin-1. Neither the monovalent (at $250 \mu \mathrm{M}$ ) nor the divalent compounds (at $25 \mu \mathrm{M}$ ) had a detectable interaction with the immobilized galectin-1 (Fig. S3, ESI $\dagger$ ).

Since we did not succeed in obtaining crystal structures of LecA complexed with the divalent inhibitors, we carried out modeling on pairs of para- and meta-compounds, selecting the high-affinity binder C5m and its para-counterpart C5p; and the longer, less active $\mathbf{E 5} \boldsymbol{m}$ and $\mathbf{E 5} \boldsymbol{p}$. The dynamics of these four compounds were simulated in the free state and in the modeled complex with LecA. In the free state, the ligands adopted a broad range of semiextended to fully extended conformations characterized by Gal:C1 - . Gal:C1 distances ranging from 20 to $31 \AA$ at a cutoff of $5 \%$ frequency (Fig. 4a, solid curves). A distance close to the crystallographic value of $29 \AA$ (PDB: 5d21) is desired for optimal divalent binding of LecA. In the longer compounds, E5m and E5p, there was a small population (around 5\%) of folded conformations (see the peaks at $5 \AA$ in Fig. 4a). The unfolding of these conformations prior to LecA binding may be in part responsible for their slower on-rate $\left(k_{\mathrm{on}}\right)$.

The four ligands modeled in complex with LecA showed a narrow distribution of the Gal:C1 $\cdots$ Gal:C1 distances (Fig. 4a, dashed lines). C5m, C5 $\boldsymbol{p}$ and E5 $\boldsymbol{p}$ sampled distances of $28.9 \pm$ $0.4 \AA, 28.1 \pm 0.9 \AA$, and $28.2 \pm 0.7 \AA$, respectively, close to the $29 \AA$ observed in crystal structures, thus indicating that the linker lengths are well-suited to bridge two LecA monomers. The narrow range for C5m (28-31 $\AA$ ) may explain the lower affinity of shorter B5m. In contrast, C5 $\boldsymbol{p}$ and E5 $\boldsymbol{p}$ have larger range (26-31 $\AA$ ) and shortening is beneficial such as in B5p. E5m with LecA displayed a higher mean distance of $30.2 \pm 0.8 \AA ̊$ (range of 28 to $33 \AA$ ). Such longer meta linker pushes the two LecA monomers slightly apart, which is not favourable. The T-shaped $\mathrm{CH}-\pi$ interaction between His50 and the phenyl aglycon was observed with higher frequency for the meta compounds C5m and E5m compared to their para analogues C5p and E5p (Fig. $4 \mathrm{~b}$ ). On the opposite, the para ligands, $\mathbf{C 5} \boldsymbol{p}$ and E5p , preferentially adopted an inverted V-shape (Fig. 4c and d) in which their phenyl aglycons sampled a variety of arrangements with respect to His50 (parallel, diagonal, T-shape).

To conclude, we designed and synthesized highly potent divalent LecA inhibitors in four linear chemical steps from

Table 1 Affinity and kinetic analyses of the LecA-inhibitor interactions determined by SPR and ITC ${ }^{a}$

\begin{tabular}{|c|c|c|c|c|c|c|c|c|c|}
\hline & \multicolumn{4}{|l|}{ meta series } & & \multicolumn{4}{|l|}{ para series } \\
\hline & $k_{\mathrm{on}}\left(\times 10^{3} \mathrm{M}^{-1} \mathrm{~s}^{-1}\right)$ & $k_{\text {off }}\left(\times 10^{-3} \mathrm{~s}^{-1}\right)$ & $K_{\mathrm{d}}(\mathrm{nM})$ & r.p. & & $k_{\mathrm{on}}\left(\times 10^{3} \mathrm{M}^{-1} \mathrm{~s}^{-1}\right)$ & $k_{\text {off }}\left(\times 10^{-3} \mathrm{~s}^{-1}\right)$ & $K_{\mathrm{d}}(\mathrm{nM})$ & r.p. \\
\hline $\mathbf{A 5 m}$ & - & - & $\begin{array}{l}4900 \pm 100 \\
2700 \pm 1300^{b}\end{array}$ & 1 & $\mathbf{A 5 p}$ & - & - & $\begin{array}{l}5600 \pm 300 \\
6100 \pm 500^{b}\end{array}$ & 1 \\
\hline B5m & $196 \pm 6$ & $5.34 \pm 0.28$ & $27.3 \pm 1.6$ & 90 & B5p & $152 \pm 3$ & $1.64 \pm 0.13$ & $10.8 \pm 1.0$ & 259 \\
\hline C5m & $59 \pm 3$ & $1.11 \pm 0.08$ & $18.9 \pm 1.6$ & 130 & $\mathrm{C} 5 p$ & $114 \pm 2$ & $2.33 \pm 0.02$ & $20.5 \pm 0.2$ & 137 \\
\hline D5m & $120 \pm 41$ & $2.67 \pm 0.38$ & $23.8 \pm 7.1$ & 103 & D5p & $121 \pm 9$ & $2.57 \pm 0.20$ & $21.4 \pm 1.0$ & 131 \\
\hline $\mathbf{E} 5 m$ & $45 \pm 4$ & $3.64 \pm 0.35$ & $80.7 \pm 11.4$ & 30 & $\mathrm{E} 5 p$ & $103 \pm 2$ & $2.19 \pm 0.45$ & $22.5 \pm 9.0$ & 124 \\
\hline F5m & $104 \pm 53$ & $3.39 \pm 1.5$ & $33.4 \pm 2.3$ & 73 & $\mathbf{F} 5 p$ & $79 \pm 5$ & $3.98 \pm 0.16$ & $50.1 \pm 1.2$ & 56 \\
\hline
\end{tabular}

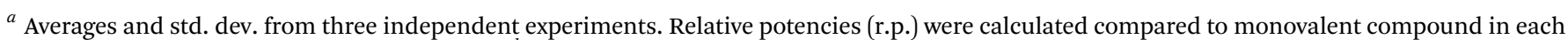
series (A5m and $\mathbf{A 5} \boldsymbol{p}$ ) and valency-normalized. ${ }^{b}$ ITC determination. 

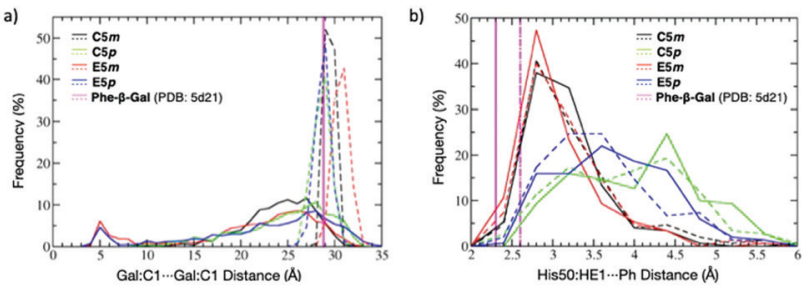

c)
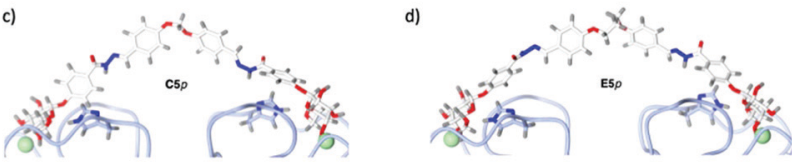

e)
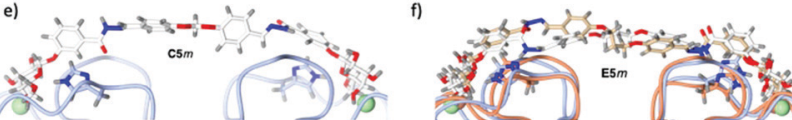

Fig. 4 Molecular dynamics simulations of $\mathrm{C} 5 \mathrm{~m}, \mathrm{E} 5 \mathrm{~m} ; \mathrm{C} 5 \mathrm{p}$; E5p: (a) distribution of Gal:C1...Gal:C1 distances of compounds in the free state (solid lines) and in complex with LecA (dashed lines). (b) Distribution of distances between His50 and the phenyl aglycon of ligands in complex with LecA (solid lines for LecA protein chain A, dashed lines for chain B). (c-f) Snapshot from trajectories of C5p (c), E5p (d), C5m (e), and two snapshots of E5m (f) indicating the conformational change of LecA.

galactose pentaacetate. These simple and rapidly accessible divalent inhibitors B5m-F5m and B5p-F5p have comparable or superior activity to the previously reported and structurally complex di- and multivalent LecA ligands. Monovalent analogs A5 $\boldsymbol{m}$ and A5 $\boldsymbol{p}$ showed binding to LecA in SPR and ITC experiments in the low micromolar range $\left(K_{\mathrm{d}}=2.7-6.1 \mu \mathrm{M}\right)$. Divalent display of these epitopes in B5m-F5m and B5p-F5p boosted binding affinity with LecA to low nanomolar values. Molecular dynamics simulations gave insights into the interplay of linker geometry and length for an optimal divalent binding. To the best of our knowledge, compound B5p with a $K_{\mathrm{d}}$ of $10.8 \mathrm{nM}$ is the most potent divalent LecA ligand reported to date with confirmed selectivity for LecA over galectin-1. Due to the simplicity of our synthetic design and readily accessible building blocks, further fine tuning and optimization of drug-like properties can be readily implemented. Future optimization of these compounds targeting LecA may provide a treatment of biofilm-associated $P$. aeruginosa infections.

The authors are grateful to Dirk Hauck (HIPS) for excellent technical assistance and Varsha R. Jumde (HIPS) for assisting with DCC. M. L. acknowledges a EU H2020 Marie Sklodowska-Curie grant (795605). Computations were run on GRICAD infrastructure, HPC-EUROPA3 project (H2020-INFRAIA-2016-1-730897), and EPCC at the University of Edinburgh, Scotland, and HPC resources from GENCI-IDRIS (Grant 2019-A0070711040). A. T. thanks the European Research Council (ERC Starting Grant, Sweetbullets) and Deutsche Forschungsgemeinschaft (Ti756/5-1). S. Y. acknowledges a RISE fellowship, German Academic Exchange Service DAAD. The work was supported by the ANR/DFG FrenchGerman GLYCOMIME project (ANR-AAPG-2017, DFG Ti756/5-1). A. I. and S. K. acknowledge support from Glyco@Alps (ANR-15IDEX02), Labex Arcane/CBH-EUR-GS (ANR-17-EURE-0003). A. K. H. H. gratefully acknowledges the ERC Starting Grant (757913).

\section{Conflicts of interest}

There are no conflicts to declare.

\section{Notes and references}

1 WHO, https://www.who.int/news-room/detail/27-02-2017-who-publisheslist-of-bacteria-for-which-new-antibiotics-are-urgently-needed, accessed 9 April 2020.

2 S. Wagner, R. Sommer, S. Hinsberger, C. Lu, R. W. Hartmann, M. Empting and A. Titz, J. Med. Chem., 2016, 59, 5929-5969.

3 A. E. Clatworthy, E. Pierson and D. T. Hung, Nat. Chem. Biol., 2007, 3, 541-548.

4 M. B. Calvert, V. R. Jumde and A. Titz, Beilstein J. Org. Chem., 2018, 14, 2607-2617.

5 J. Meiers, E. Siebs, E. Zahorska and A. Titz, Curr. Opin. Chem. Biol., 2019, 53, 51-67.

6 S. Cecioni, A. Imberty and S. Vidal, Chem. Rev., 2015, 115, 525-561.

7 K. Winzer, C. Falconer, N. C. Garber, S. P. Diggle, M. Camara and P. Williams, J. Bacteriol., 2000, 182, 6401-6411.

8 S. P. Diggle, R. E. Stacey, C. Dodd, M. Cámara, P. Williams and K. Winzer, Environ. Microbiol., 2006, 8, 1095-1104.

9 D. Tielker, S. Hacker, R. Loris, M. Strathmann, J. Wingender, S. Wilhelm, F. Rosenau and K. E. Jaeger, Microbiology, 2005, 151, 1313-1323.

10 J. Rodrigue, G. Ganne, B. Blanchard, C. Saucier, D. Giguère, T. C. Shiao, A. Varrot, A. Imberty and R. Roy, Org. Biomol. Chem., 2013, 11, 6906-6918.

11 I. Joachim, S. Rikker, D. Hauck, D. Ponader, S. Boden, R. Sommer, L. Hartmann and A. Titz, Org. Biomol. Chem., 2016, 14, 7933-7948.

12 R. Sommer, S. Wagner, K. Rox, A. Varrot, D. Hauck, E. C. Wamhoff, J. Schreiber, T. Ryckmans, T. Brunner, C. Rademacher, R. W. Hartmann, M. Brönstrup, A. Imberty and A. Titz, J. Am. Chem. Soc., 2018, 140, 2537-2545.

13 R. Sommer, K. Rox, S. Wagner, D. Hauck, S. S. Henrikus, S. Newsad, T. Arnold, T. Ryckmans, M. Brönstrup, A. Imberty, A. Varrot, R. W. Hartmann and A. Titz, J. Med. Chem., 2019, 62, 9201-9216.

14 S. Wagner, D. Hauck, M. Hoffmann, R. Sommer, I. Joachim, R. Müller, A. Imberty, A. Varrot and A. Titz, Angew. Chem., Int. Ed., 2017, 56, 16559-16564.

15 R. U. Kadam, D. Garg, J. Schwartz, R. Visini, M. Sattler, A. Stocker, T. Darbre and J.-L. Reymond, ACS Chem. Biol., 2013, 8, 1925-1930.

16 M. Mammen, S.-K. Choi and G. M. Whitesides, Angew. Chem., Int. Ed., 1998, 37, 2754-2794.

17 K. Drickamer and M. E. Taylor, Curr. Opin. Struct. Biol., 2015, 34, 26-34.

18 A. Bernardi, J. Jiménez-Barbero and A. Casnati, et al., Chem. Soc. Rev., 2013, 42, 4709-4727.

19 G. Cioci, E. P. Mitchell, C. Gautier, M. Wimmerová, D. Sudakevitz, S. Pérez, N. Gilboa-Garber and A. Imberty, FEBS Lett., 2003, 555, 297-301.

20 J. E. Gestwicki, C. W. Cairo, L. E. Strong, K. A. Oetjen and L. L. Kiessling, J. Am. Chem. Soc., 2002, 124, 14922-14933.

21 F. Pertici and R. J. Pieters, Chem. Commun., 2012, 48, 4008-4010.

22 G. Yu, A. C. Vicini and R. J. Pieters, J. Org. Chem., 2019, 84, $2470-2488$.

23 R. Visini, X. Jin, M. Bergmann, G. Michaud, F. Pertici, O. Fu, A. Pukin, T. R. Branson, D. M. E. Thies-Weesie, J. Kemmink, E. Gillon, A. Imberty, A. Stocker, T. Darbre, R. J. Pieters and J. L. Reymond, ACS Chem. Biol., $2015,10,2455-2462$

24 A. Novoa, T. Eierhoff, J. Topin, A. Varrot, S. Barluenga, A. Imberty, W. Römer and N. Winssinger, Angew. Chem., Int. Ed., 2014, 53, 8885-8889.

25 S.-F. Huang, C.-H. Lin, Y.-T. Lai, C.-L. Tsai, T.-J. R. Cheng and S.K. Wang, Chem. - Asian J., 2018, 13, 686-700.

26 M. Bergmann, G. Michaud, R. Visini, X. Jin, E. Gillon, A. Stocker, A. Imberty, T. Darbre and J. L. Reymond, Org. Biomol. Chem., 2016, 14, $138-148$

27 M. Mondal and A. K. H. Hirsch, Chem. Soc. Rev., 2015, 44, $2455-2488$.

28 A. M. Hartman, R. M. Gierse and A. K. H. Hirsch, Eur. J. Org. Chem., 2019, 3581-3590.

29 I. Cumpstey, E. Salomonsson, A. Sundin, H. Leffler and U. J. Nilsson, ChemBioChem, 2007, 8, 1389-1398. 